\title{
Consensus statement for inert gas washout measurement using multiple- and single-
} breath tests

\begin{abstract}
Paul D. Robinson, Philipp Latzin, Sylvia Verbanck, Graham L. Hall, Alexander Horsley, Monika Gappa, Cindy Thamrin, Hubertus G.M. Arets, Paul Aurora, Susanne I. Fuchs, Gregory G. King, Sooky Lum, Kenneth Macleod, Manuel Paiva, Jane J. Pillow, Sarath Ranganathan, Felix Ratjen, Florian Singer, Samatha Sonnappa, Janet Stocks, Padmaja Subbarao, Bruce R. Thompson and Per M. Gustafsson
\end{abstract}

ABSTRACT: Inert gas washout tests, performed using the single- or multiple-breath washout technique, were first described over 60 years ago. As measures of ventilation distribution inhomogeneity, they offer complementary information to standard lung function tests, such as spirometry, as well as improved feasibility across wider age ranges and improved sensitivity in the detection of early lung damage. These benefits have led to a resurgence of interest in these techniques from manufacturers, clinicians and researchers, yet detailed guidelines for washout equipment specifications, test performance and analysis are lacking. This manuscript provides recommendations about these aspects, applicable to both the paediatric and adult testing environment, whilst outlining the important principles that are essential for the reader to understand. These recommendations are evidence based, where possible, but in many places represent expert opinion from a working group with a large collective experience in the techniques discussed.

Finally, the important issues that remain unanswered are highlighted. By addressing these important issues and directing future research, the hope is to facilitate the incorporation of these promising tests into routine clinical practice.

KEYWORDS: Adult, lung function, monitoring, paediatric, validation

\section{CONTENTS}

Introduction

Mechanisms of ventilation distribution inhomogeneity

SBW and MBW tests

Equipment specifications

Validation of washout equipment

Suitability of current washout systems across age groups

Outcomes

Functional residual capacity

Measures of ventilation distribution inhomogeneity

Trapped gas volume

Closing volume and closing capacity

Impact of inert gas choice

Acceptability criteria for testing
AFFILIATIONS

For a full list of author affiliations, please refer to the Acknowledgements.

CORRESPONDENCE

P.D. Robinson

Dept of Respiratory Medicine

The Children's Hospital at Westmead Locked Bag 4001

Westmead

Sydney

NSW 2145

Australia

E-mail:dr.pdrobinson@gmail.com

Received:

May 022012

Accepted after revision:

Sept 072012

First published online:

Feb 072013

After publication of the erratum in the November 2013 issue of the European Respiratory Journal, the online version of this article has been revised. 
Multiple-breath washout . . . . . . . . . . . . . . . . . . 516

Test termination . . . . . . . . . . . . . . . . 518

FRC repeatability . . . . . . . . . . . . . . . . . . . . 518

\section{INTRODUCTION}

The architecture of the airway tree promotes even distribution and optimal mixing of inhaled gas with resident gas. Multiplebreath and single-breath inert gas washout tests (MBW and SBW, respectively) assess the efficiency of ventilation distribution [1, 2]: in principle, efficiency of inert marker gas clearance from the lungs, or gas mixing within the time frame of a single breath, respectively. Suitable inert gases must be safe to inhale at the concentrations used, not participate in gas exchange, and not dissolve significantly in blood or other tissues. Options include both endogenous (nitrogen: $\mathrm{N}_{2}$, and argon) and exogenous gases (sulfur hexafluoride: $\mathrm{SF}_{6}$, helium: $\mathrm{He}$, and methane). Marked ventilation distribution abnormalities occur in obstructive lung disease [3,4] despite normal ventilatory capacity as measured by spirometry [5-10]. Washout tests may provide insight into mechanisms behind abnormal ventilation distribution and localisation of pathology. MBW is particularly attractive as it uses either relaxed tidal breathing (mostly in paediatric settings) or a fixed tidal volume (usually $1 \mathrm{~L}$ in
Single-breath washout . . . . . . . . . . . . . . . 518

Future work and conclusions . . . . . . . . . . 518

References ... . . . . . . . . . . . . . . . . . . 520

adults) without need for maximal effort, thereby offering feasibility in all age groups [5, 7, 9, 11-14], driving recent strong paediatric interest. Despite this and unique insights into disease onset, widespread clinical use has yet to be achieved and further work that is required is limited by a lack of carefully validated robust commercial washout systems.

Washout recording systems determine inspired and expired inert gas volumes, by continuously measuring inert gas concentrations synchronised with respiratory flow. The overall aims of this standardisation document are to promote and facilitate use of open-circuit washout systems (i.e. minimal rebreathing of expired air), and achieve quality assured results, comparable between laboratories, using validated systems suitable across age groups and disease conditions. This paper is directed to manufacturers, researchers, clinicians and respiratory technicians. Recommendations are made for testing infants, children and adults, reflecting broad clinical and research interest. Application in different age groups may require age-specific modifications, assumptions and limitations.

\section{TABLE 1 Key recommendations from this standardisation document}

Recommendations contained in this document are based on evidence where available. If no evidence exists, the recommendations are based on expert opinion, and will continue to evolve over time and be updated in future documents as further insight is gained.

SBW and MBW testing offer complimentary information, but the choice of test used may be age and disease dependent. Depending on the pathology under study, relationships between MBW-derived indices may help identify the type of structural changes.

A series of individual equipment component recommendations are provided in this document. It is, however, unlikely that all individual criteria outlined will be fulfilled by any one system, which is why overall system performance during validation and subsequent testing is the central aspect of importance.

FRC measurement validation is an essential step and should assess all the stages of the measurement including post-data acquisition processing procedures, such as BTPS correction. FRC measurement accuracy does not ensure accuracy of all other derived indices and biological control measurements and monitoring is essential.

Responsibility for commercial system validation and ongoing reliability of system performance should lie with the manufacturer. However, close vigilance by the end user is essential. Biological control measurement and monitoring during subsequent clinical and research testing is an essential component of this.

FRC and ventilation inhomogeneity indices must relate to the same geometric reference point in the airstream. FRC end-point for measurement during the washout should correspond to the end of test threshold used for ventilation inhomogeneity index analysis, e.g. LCl threshold.

The method of FRC determination, indices of ventilation distribution inhomogeneity calculation, and any corrections performed (e.g. VT or VC) must be clearly described. Both corrected and uncorrected values should be reported to facilitate a priori analysis in the future.

Suitability of open-system inert gas washout equipment for use in different age ranges is determined by the overall contribution of characteristics such as equipment dead space and analyser dynamic properties.

The choice of inert gas used is dependent on many factors, but impacts on the results obtained. Normative values are inert gas specific. Comparison of multiple simultaneously measured inert gases may provide additional information about the location of underlying pathology. Correction for tissue $\mathrm{N}_{2}$ diffusion into the lung is not currently recommended due to a lack of appropriate data to base corrections on.

A variety of factors may lead to differences in reported washout indices between centres and experimental conditions under which normative data are obtained should be clearly described.

Quality control during testing is critical and extends beyond equipment performance and software feedback to also include close observation by the operator of the subject's behaviour during testing and how this affects the data obtained. Adequate operator training and appreciation of all factors influencing test results is essential.

Breathing patterns during testing should be kept similar between subjects to facilitate comparison of results. In adults this is achieved by using strict breathing regimens where feasible and in younger children (aged $\leqslant 16 \mathrm{yrs}$ ) by distraction to encourage relaxed tidal breathing.

The end test threshold used for MBW tests will depend on the ventilation distribution index (or indices) being reported.

Formal FRC repeatability criteria for MBW indices should not be routinely applied, but FRC values within $10 \%$ should be viewed as encouraging. FRC values differing by more than $25 \%$ from the median of three test values should be excluded.

SBW: single-breath washout; MBW: multiple-breath washout; FRC: functional residual capacity; BTPS: body temperature, ambient pressure, saturated with water; LCI: lung clearance index; $\mathrm{V} T_{\mathrm{T}}$; tidal volume; $\mathrm{VC}$ : vital capacity; $\mathrm{N}_{2}$ : nitrogen gas. 


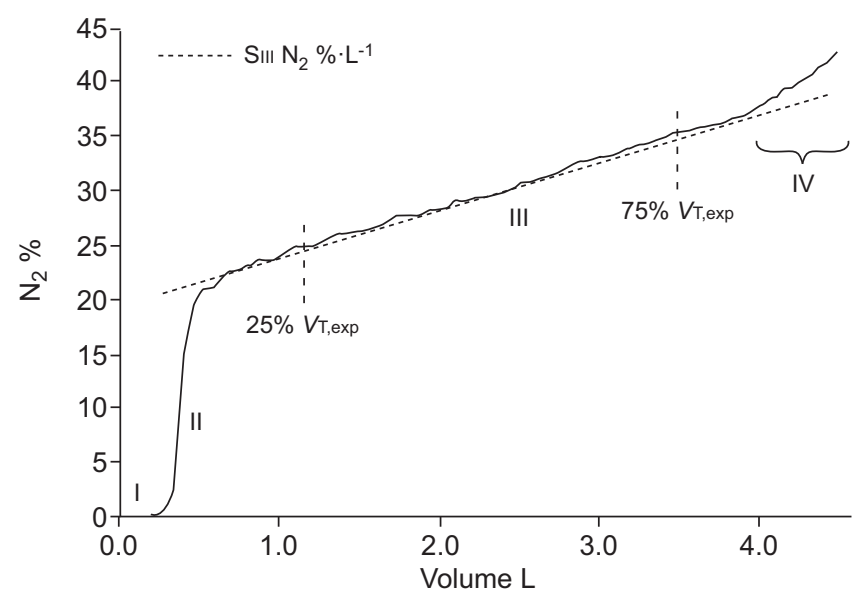

FIGURE 1. Example of a typical single-breath washout (SBW) trace. Nitrogen gas $\left(\mathrm{N}_{2}\right)$ expirogram showing calculation of phase III slope (SIII) in a vital capacity SBW test in a 60 -yr-old smoker. SIII is calculated between $25 \%$ and $75 \%$ of the expired volume (SIII $4.4 \% \cdot \mathrm{L}^{-1}$ ), to avoid the contribution of phase IV. The four phases of the expirogram are also demonstrated: phase I (absolute dead space), phase II (bronchial phase), phase III (alveolar phase) and phase IV (fast rising phase at end of expiration). Closing volume (CV) is the expired volume (L) from the start of the upward deflection where phase IV starts, to the end of the breath. If residual volume (RV) is known, closing capacity (CC) can be calculated: $C C=C V+R V . V_{T}$,exp: expired tidal volume.

Specific aims of this document are to: 1) describe the principles and physiological concepts behind MBW and SBW tests; 2) outline equipment requirements, appropriate system quality control and validation; 3) describe available washout outcomes, factors influencing their calculation, and insights provided into underlying mechanisms of ventilation distribution inhomogeneity; 4) provide recommendations and test acceptability criteria in different age groups; and 5) highlight important future research.

Recommendations will continue to evolve as further insight is gained. Clinical utility has been summarised elsewhere [15-19]. Key recommendations are summarised in table 1.

\section{MECHANISMS OF VENTILATION DISTRIBUTION INHOMOGENEITY}

Ventilation distribution occurs by convection and diffusion [20]. Three principal mechanisms generate inhomogeneity [21]. 1) Convection-dependent inhomogeneity (CDI) in the conducting airway zone (i.e. airways proximal to terminal bronchioles) [22]. 2) Diffusion-limitation related inhomogeneity in pathologically enlarged acinar structures (rare). 3) Interaction between convection and diffusion in an intermediate zone at the level of the diffusion-convection front.

In adult healthy lungs, this quasi-stationary diffusion-convection front, which determines where these mechanisms can operate, is thought to arise around the acinar entrance [23]. Inhomogeneity of ventilation distribution is reflected in delayed MBW markergas clearance, raised SBW phase III slope (SIII), explained in figure 1, and magnitude and progression of MBW concentration normalised phase III slopes (SnII) through subsequent breaths (fig. 2); in the latter, SIII normalisation by expired alveolar inert gas concentration is required to compare progression.
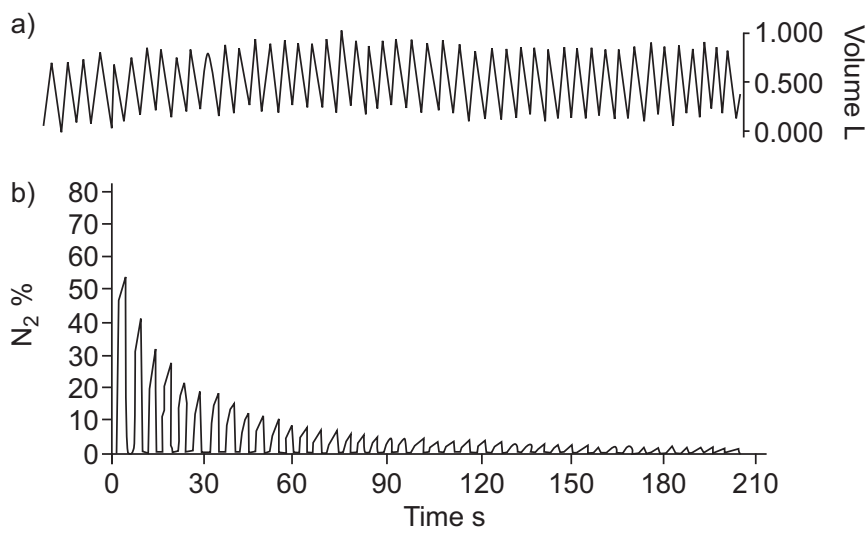

FIGURE 2. Example of a typical multiple-breath washout (MBW) trace. Time series display of a) volume and b) nitrogen gas $\left(N_{2}\right)$ from an $N_{2}$ MBW test in a female aged 15 yrs with cystic fibrosis. Stable breathing and end-tidal inert gas concentration are seen prior to commencing the washout phase.

CDI results from differences in specific ventilation between lung units sharing branch points in the conducting airway zone in combination with sequential filling and emptying among these units [24]. CDI contributes to increased SIII in SBW and generates a continuous rise in SnIII through subsequent MBW breaths [25]. Diffusion convection-interaction-dependent inhomogeneity (DCDI), which occurs in the region of the acinar entrance, increases SnIII if structural asymmetry is present at branch points (e.g. differences in cross-sectional area and/or subtended lung volumes). In normal adult lungs, DCDI is the major contributor to SBW SIII [24] and DCDI contribution to MBW SnIII reaches its maximum at approximately five breaths [25].

\section{SBW AND MBW TESTS}

SBW and MBW assess ventilation distribution inhomogeneity at differing lung volumes. The most widely used is the $\mathrm{N}_{2} \mathrm{SBW}$ test [1], which involves a vital capacity (VC) manoeuvre performed at low constant flow $\left(400-500 \mathrm{~mL} \cdot \mathrm{s}^{-1}\right)$ : exhalation to residual volume $(\mathrm{RV})$, inhalation of $100 \%$ oxygen gas $\left(\mathrm{O}_{2}\right)$ to total lung capacity (TLC), then washout during exhalation from TLC to RV $[1,26]$, where SIII is measured over the mid portion of the expirogram (fig. 1). For exogenous inert gas SBW, the inert gas is washed in during inhalation from RV to TLC, before washout during exhalation to RV. VC SBW SIII is influenced to a greater degree by gravitational and nongravitational inter-regional differences in gas distribution and airway closure during the inspiratory phase [27-29], compared to tidal breathing protocols. Actual peripheral airway contribution to VC SBW SIII is uncertain. Modification by initial wash-in from functional residual capacity (FRC) to either TLC or a volume above FRC (e.g. 1 L) [30], better reflects inhomogeneity present during near-tidal breathing and may be a more sensitive index of peripheral airway involvement [31].

MBW assesses ventilation distribution inhomogeneity during tidal breathing from FRC, by examining inert gas clearance over a series of breaths. Exogenous gas washout requires an initial wash-in phase. MBW requires only passive cooperation and minimal coordination, but is more time consuming. It appears to be the most informative of these tests. In contrast to MBW, SBW SIII using a single inert gas does not separate CDI 
and DCDI contributions, though some information about location of pathological processes may be gained by comparing simultaneous SBW SIII of inert gases with widely different molecular mass (as described in the section entitled Impact of inert gas choice). SBW may be sufficient for some patient groups: in patients for whom DCDI is thought to be the main mechanism, SBW initiated from FRC approximates the first tidal expiration of a MBW, which contains most of the DCDI information. Studies directly comparing SBW and MBW are rare or non-existent.

\section{EQUIPMENT SPECIFICATIONS}

Key components and principles exist when designing washout devices (fig. 3). Individual component recommendations are summarised in table 2 and section E2 in the online supplementary material. It is unlikely that all individual criteria outlined will be fulfilled by any one system, which is why overall system performance during validation and subsequent testing is the central aspect (table 3). Recommendations for online and offline washout software are summarised in tables 4 and 5 .

Accurately measured flow and inert gas concentration must be meticulously synchronised. Asynchrony between flow and gas signals in real-time measurement is due to gas sample transit time from airstream to inert gas analyser and/or gas analyser response time. Inert gas concentration measurements should ideally occur across the mainstream to minimise the error introduced by streaming, and be synchronous with flow signal. Mainstream gas analysers generally have shorter rise times than sidestream analysers but may introduce additional equipment deadspace, which in turn may have detrimental effects on ventilation during testing. Short analyser rise times become increasingly important as breathing rate increases, such as in young infants. Overall contribution of characteristics such as these determines suitability for different age ranges, as illustrated by the detailed discussion of current published systems as shown in section E2.7 in the online supplementary material.

\section{VALIDATION OF WASHOUT EQUIPMENT}

Recommended washout equipment validation is FRC measurement accuracy: FRC values within $5 \%$ of known volume for at least $95 \%$ of values [32] across the range of lung volumes, $V \mathrm{~T}$ and respiratory rates encountered during subsequent clinical testing [34, 35]. Validation should assess all stages of measurement including post-data acquisition processing procedures, such as body temperature, ambient pressure, saturated with water (BTPS) correction. Recently, optimised lung model design [36] has incorporated simulated BTPS conditions for validation of both established and emerging MBW systems (fig. 4) [35] and is the recommended approach. Validation should be repeated if significant changes in hardware or

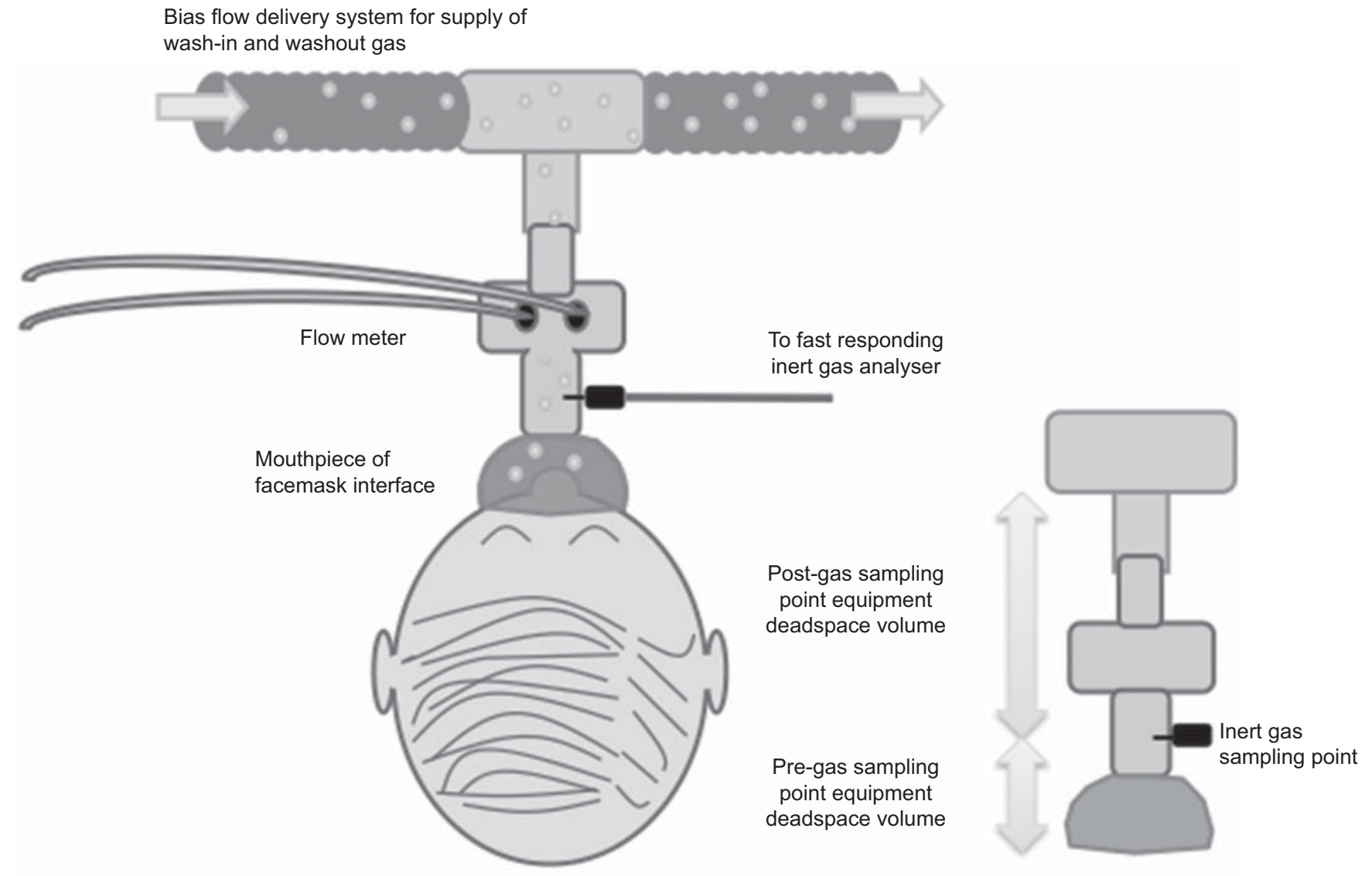

FIGURE 3. Schematic illustration of a generic inert gas washout system. The figure illustrates a generic washout system. Hardware required for washout is relatively simple: a flow meter, a fast responding inert gas analyser, a gas delivery system and a patient interface. The equipment-related deadspace volume (VD) can be divided into pre- and postgas sampling points. Post-gas sampling point VD effectively introduces a small rebreathing chamber. Pre-gas sampling point $V D$ is an extension of anatomical $V D$. 


\section{TABLE 2 Summary of component recommendations for inert gas washout system characteristics}

\begin{tabular}{|c|c|}
\hline Flow measurement & $\begin{array}{l}\text { Instantaneous flow accuracy within } 5 \% \text { across the range of flows encountered during clinical testing and volume accuracy } \\
\text { within } 3 \% \text { using a precision calibration syringe. }\end{array}$ \\
\hline Sample flow & $\begin{array}{l}\text { Ideally, all side-stream washout systems should correct for sample flow. If not performed or achievable, sample flow } \\
\text { should be minimised: }<20 \mathrm{~mL} \cdot \mathrm{min}^{1} \text { for paediatric and }<40 \mathrm{~mL} \cdot \mathrm{min}^{-1} \text { for adult apparatus where gas sample point is } \\
\text { proximal to flow metre. }\end{array}$ \\
\hline Gas analyser accuracy & $\begin{array}{l}\text { Linearity within } 1 \% \text { relative of full scale (e.g. } 0-80 \% \text { is } \pm 0.8 \% \text { at } 80 \% \mathrm{~N}_{2} \text { ) to ensure appropriate assessment of starting } \\
\text { concentration, and within } 5 \% \text { relative of any lower value (e.g. } 0.25 \% \text { at } 5 \% \mathrm{~N}_{2} \text { ) down to } 1 / 40 \text { of the starting concentration. } \\
\text { Initial assessment should incorporate both dry and humid conditions. Monitor gas analyser accuracy, stability and linearity } \\
\text { annually using at least three reference points of gas concentration. }\end{array}$ \\
\hline Equipment-related dead space & $\begin{array}{l}\text { Total equipment deadspace for young children should be }<2 \mathrm{~mL} \cdot \mathrm{kg}^{-1} \text { bodyweight, and ideally }<1 \mathrm{~mL} \cdot \mathrm{kg}^{-1} \text { in infants. } \\
\text { Recommendations should be adhered to in older subjects, until further evidence is available. An upper limit of } 70 \mathrm{~mL} \\
\text { should be adhered to for adults including hygiene filters if used. }\end{array}$ \\
\hline Equipment-related resistance & Should be minimised for both inspiration and expiration to avoid effects on breathing pattern and FRC during test. \\
\hline
\end{tabular}

The table is expanded with further explanation in section E2.6 in the online supplementary data. $\mathrm{N}_{2}$ : nitrogen gas; FRC: functional residual capacity.

software algorithms occur [39]. All MBW ventilation inhomogeneity indices depend on accurate FRC determination, but FRC validation alone may not be sufficient to ensure accuracy of derived ventilation distribution indices. During subsequent clinical or research testing, biological controls should monitor measurement stability (e.g. three to four healthy staff members performing $\mathrm{MBW}$ in triplicate, monthly). Marked variation beyond normal observed pattern should prompt further careful evaluation of device performance and procedures.

A variety of factors may generate differences in reported indices between centres (table 6), and until standardisation is achieved, normative data is at best tentative and likely to be inert gas, equipment and software specific. Experimental conditions under which normative data are obtained should be clearly described in manuscripts.

\section{SUITABILITY OF CURRENT WASHOUT SYSTEMS ACROSS AGE GROUPS}

The only current system applicable across all age groups is custom built and based on the respiratory mass spectrometer (RMS). RMS is the current gold standard gas analyser offering simultaneous measurement of multiple gases in constant conditions, full linearity, low sample flow and short response time [39]. This custom washout system exists in several centres [5, 7, 40-42], but may be too expensive and impractical for widespread use.

In MBW using $\mathrm{N}_{2}$, inhalation of $100 \% \mathrm{O}_{2}$ may alter breathing patterns in infants [43] and subsequent MBW outcomes, but impact on breathing pattern beyond infancy is considered minimal. As an alternative to emission spectrophotometer $\mathrm{N}_{2}$ analyser systems (requiring vacuum pumps), indirect $\mathrm{N}_{2}$ measurement systems have been proposed based on simultaneous $\mathrm{O}_{2}$ and $\mathrm{CO}_{2}$ measurement [35] or changes in molar mass (MM) [34] (see section E3 in the online supplementary material). Potential for additive errors with indirect measurement places even greater emphasis on adequate quality control.

$\mathrm{MM}$ based measurement of $\mathrm{SF}_{6}$ or He are also feasible [44-46]. Mainstream $\mathrm{MM} \mathrm{SF}_{6}$ washout has been validated in infants [46, 47]; however, lack of validated correction algorithms for detrimental temperature and humidity fluctuations limit utility beyond infancy [48]. Sidestream MM washout incorporating Nafion ${ }$ tubing to stabilise temperature and humidity [49] has been validated for older age groups [38, 50], but current equipment deadspace volume $(V D)$ precludes use in infancy.

Modified photoacoustic analyser based systems have been validated for use in adults and school age children $[9,51]$, but are not currently commercially available. Feasibility into younger ages will depend on minimisation of longer analyser response times. Detrimental impact of high sample flow used in these systems on measured flows may be reduced by gas sensor placement distal to flow measurement, but requires careful evaluation. Sampling bias flow gas during low expiratory flows must also be avoided. The commercially available photoacoustic analyser based closed circuit system is not discussed in this manuscript [52].

\section{OUTCOMES}

\section{Functional residual capacity}

FRC measured by MBW (FRCgas) represents the volume of lung gas, at end expiration (assessed at the breath immediately preceding washout), in direct communication with the airway opening, excluding gas trapped in lung regions not ventilated by 


\section{TABLE 3 Overall recommendations for washout systems}

Instantaneous flow within 5\%, and $V T$ and CEV measurement accuracy within $3 \%$.

Quality of gas signals allowing determination of FRC, end-tidal gas concentration and SIII down to $1 / 40$ of the starting inert-gas concentration with sufficient accuracy and resolution (see below)

FRC measurement accuracy within $5 \%$ of the true FRC value (for $95 \%$ of values), using a realistic lung model incorporating BTPS conditions across the intended volume range and breathing pattern of the system. Commercial systems manufacturers should perform this validation both prior to sale and whenever significant hardware or software modifications are made to existing devices. Re-evaluation should be performed as necessary if marked variation occurs beyond the normal observed pattern for biological controls during clinical research use.

The static and dynamic properties of the gas analyser (accuracy, response time and signal-to-noise ratio) should ensure a linear and accurate gas signal. End-tidal inert-gas concentrations should be within $1 \%$ relative to inert-gas concentrations at the start and $5 \%$ relative to inert-gas concentrations at the end of the washout (i.e. at $1 / 40$ of the starting concentration).

The manufacturers of commercial inert-gas washout systems should demonstrate these features prior to commercial release, with data being included within supporting documentation. VT: tidal volume; CEV: cumulative expired volume; FRC: forced residual capacity; SIII: phase III slope; BTPS: body temperature, ambient pressure saturated with water.

tidal breaths. FRCgas is, therefore, often lower than plethysmographic FRC, especially in obstructive lung disease [53]. FRCgas $=V \mathrm{IG} / \mathrm{Cet}, \mathrm{IG}$ (initial-final), where: $V \mathrm{IG}$ is net volume of inert gas expired, and Cet is end-tidal concentration of inert gas. VIG is the sum of the integral products of exhaled flow and gas concentration for each washout breath, corrected for re-inspired gas, contained within the $V \mathrm{D}$ after the post-gas sampling point (fig. 3, see section E5.2 in online supplementary material).

\section{TABLE 4 Recommendations for online washout software}

Software to display flow, volume and respiratory rate monitoring are essential for both fixed breathing protocols (SBW and MBW in adults and older adolescents) and to monitor and stabilise tidal breathing in younger subjects

Volume time series display of BTPS adjusted data should be of sufficient length and size to detect volume drift

Differentiating technical causes from physiological causes of volume drift may be difficult

Sudden step changes in volume may indicate leak

Graphical display of inert gas concentration traces both during the wash-in and washout phases

To assess suitability of timing to start the washout phase

To monitor for leaks (see table 5), this should include a clear display of the "zero" inert gas baseline concentration level, which may not be achieved in cases such as insufficient washout as supply or leak; if an automated correction of deviation from zero baseline is performed by the software, the magnitude of this deviation correction must be clearly visible to alert the user

Accurate breath detection of start and end of inspiration and expiration adhering to existing standards for identification of tidal breaths [32, 33]; these standards were developed for infants but are extendable for application in adults

Distinguishing start and end of inspirations and expirations from minor fluctuations in flow during pauses and irregular breathing is usually accomplished using flow thresholds but a combination of flow and volume based criteria may be better

Accurate detection of end-tidal inert gas concentration

Average over 5-10 samples (or 25-50 ms), ending five samples (or $25 \mathrm{~ms}$ ) before the end of expiration (see section E4.1 in the online supplementary data) Alternatively average over $95-98 \%$ of the expired volume

If SnIII progression is being measured then display the breath-by-breath inert gas expirogram to allow the user to ensure sufficient SIII is visible $(\geqslant 50 \%$ of the expired $V T)$

To aid the user in determining when end-of-test thresholds are met, online analysis should display End-tidal inert gas concentration

If SnIII progression or moment ratios are being measured: FRC and lung turnover (CEV/FRC for each breath) as the washout proceeds

To limit the time required for testing, automated calculation of the following indices should occur at the end of each test FRC

Breath-by-breath calculation and display of $V_{D}$,aw (quality control for leak detection)

Global ventilation distribution indices

Offline analysis and quality control can then be performed as required by the operator (as detailed in the section entitled Validation of washout equipment)

Warning messages should inform the operator when important quality control steps have not been fulfilled 


\section{TABLE 5 Recommendations for offline washout software}

\section{Software transparency for}

All correction algorithms and factors applied to data (e.g. BTPS and temperature modelling)

All algorithms used for subsequently calculated indices

Method used to synchronise flow and inert marker gas concentration signals

Normative data or upper limit of normal incorporated, including details of source and population characteristics (number of subjects, sex distribution, age range, ethnic group, etc.

\section{General recommendations}

Full availability of raw data, calibrated data and BTPS-converted data which should be saved and readily exportable in widely acceptable formats, e.g. ASCII (.txt) or .xls

Ability to assess accuracy of flow and inert gas concentration synchronisation, re-measure and manually adjust as necessary

Ability to review tidal volume tracing to ensure correct identification of breath detection (start and end-points), and manually adjust as necessary

Ability to review inert gas expirogram for each breath, and manual adjustment if necessary, to ensure correct estimation of

End-tidal inert marker gas concentration

SIII if SBW or if MBW SnIII analysis is being performed

Ability to examine for and correct any gas-analyser drift occurring during the test. The zero calibration point may be useful as a reference for many of the gases used ( $\mathrm{N}_{2}$, $\mathrm{CO}_{2}$,

$\mathrm{He}$ and $\mathrm{SF}_{6}$ ) whilst $100 \%$ can be used for $\mathrm{O}_{2}$. Any correction applied should be clearly stated

If available, monitor end-tidal $\mathrm{CO}_{2}$ values during MBW to screen for hyperventilation

\section{FRC}

FRC is measured over all breaths of the washout, and updated after each breath, until a defined end-point in time. The end-point used for FRC determination should

correspond to the end-test threshold used for ventilation inhomogeneity indices (e.g. LCl threshold)

Exhaled inert gas volume must be corrected for re-inspired gas from the post-gas VD for each breath

Reported FRC is that measured at the FRCgs. If other FRC values are reported, e.g. FRCao (i.e. FRCgs - pre-gas sampling point VD) these values should be described appropriately

Report mean, SD and CoV of three technically acceptable measurements

If only two technically acceptable measurements are available, report mean only, and state e.g. "based on two measurements alone"

If FRC values are not within $10 \%$ of the highest FRC value, then alert the operator. Exclude FRC values which differ by $>25 \%$ from the median FRC value across the three

tests. Excluded tests should not be used for calculation of other MBW indices

\section{Indices of global ventilation distribution inhomogeneity (e.g. $\mathrm{LCl}$ and moment ratios)}

Correct $V T$ for external VD (see section E6.2 in the online supplementary material)

Use appropriate corresponding FRC for calculation

Report mean, SD and CoV of three technically acceptable measurements

If only two technically acceptable measurements are available, report mean and \% difference, and state "based on two measurements alone"

If $\mathrm{LCl}$ values are more than $1.0 \mathrm{TO}$ apart (highest versus lowest), then alert the operator to perform further tests

\section{SnIII analysis (if performed)}

Calculation of SIII and SnIII

SIII limits set to maximise the phase III used for linear regression, excluding phase II and phase IV contributions, and be manually adjustable, typically $50-95 \%$ of the expired volume in adults and $65-95 \%$ of the expired volume in children

Manual adjustment of the SIII for breaths, where marked low frequency noise (or cardiogenic oscillations) or phase IV phenomena occur if automated estimations of SIII

Expired inert gas concentration used for SIII normalisation (e.g. mean expired concentration or mean SIII concentration) should be clearly stated

Acceptance criteria for breaths - identify and discard SnIII values of breaths that do not fulfil the following criteria

Specific to tidal breathing protocols (e.g. paediatrics)

Adequate expired volume for SnIII calculation: volume corresponding to SIII should be $>50 \%$ of expiratory VT

The expired volume should not be excessive: volume corresponding to SIII should not be $>75 \%$ of expiratory $V_{T}$

Note: to try and achieve suitable breaths, an initial tidal breathing range of $10-15 \mathrm{~mL} \cdot \mathrm{kg}^{-1}$ can be used but may need to be adjusted for the individual patient depending on the expirogram seen

Specific to adult protocols using $V_{T}$ of $1 \mathrm{~L}$

Expired volume should be $>0.950 \mathrm{~L}$

Expired volume should not be $>1.4 \mathrm{~L}$

A clear SIII should be identifiable. Failure to identify SIII due to the presence of artefact (e.g. breath hold, cardiogenic oscillations, cough) should prompt exclusion of that SnIII value

When SnIII values are excluded do not discard the contribution of that breath to other indices (e.g. FRC and TO), only the SnIII value

Tests should only contribute to overall SnIII analysis if at least two out of three of the breaths remain after SnIII breath exclusion. If $>1 / 3$ of SnIII values have been excluded due to above criteria then that entire test should be discarded

Number of excluded SnIII values and reasons for exclusion should be reported

Presentation of SnIII data

Data collated from all acceptable breaths of the three technically acceptable MBW tests

Acceptable first breath quality on all three tests for subsequent Sacin calculation

In TO calculation, FRC and VT are calculated from the same airstream reference point used in ventilation inhomogeneity indices (see the online supplementary material section E6.2)

Data displayed graphically as SnIII (y-axis) versus TO for each breath (x-axis)

SnIII and SnIII $\times$ VT (i.e. VT-corrected SnIII) displayed for each breath on two separate graphs.

These indices rely on the fact that DCDI generates a horizontal asymptote and CDI does not and are therefore only valid in cases where SnIII progression does not show a horizontal asymptote

Clinical indices calculation

Sacin calculation

Requires three technically acceptable first breath SnIII values

Sacin calculated as the mean SnIII of the three first breaths minus the Scond contribution (based on the mean TO value of the three first breaths)

Scond calculation

Scond calculated as the linear regression of SnIII values between approximately 1.5 and 6.0 TO

Calculate $95 \% \mathrm{Cl}$ of the Scond regression, reject outlying values and repeat linear regression; data should be pooled from all three runs

If SnIII analysis is performed with only two or less technically acceptable MBW tests, this should be clearly stated on the report and results interpreted with caution

SBW SIII

Report as mean, SD and CoV of three technically acceptable measurements

If only two technically acceptable measurements are available, report mean and actual difference, and state "based on two measurements"

VC measurements not within $10 \%$ of highest VC value across the SBW tests, then alert the operator

Report both SIII $\left(\% \cdot L^{-1}\right)$ and SIII $\times$ expiratory VC (\%) separately.

BTPS: body temperature, ambient pressure, saturated with water; ASCII: American Standard Code for Information Interchange; SIII: phase III slope; SBW: single-breath washout; MBW: multiple-breath washout; SnIII: normalised SIII; $\mathrm{N}_{2}$, nitrogen; $\mathrm{CO}_{2}$ : carbon dioxide; He: helium; and SF : sulfur hexafluoride; $\mathrm{O}_{2}$ : oxygen; FRC: functional residual capacity; LCl: lung clearance index; VD: deadspace volume; FRCao: FRC at the airway opening; FRCgs: FRC measured at the gas sampling point; CoV: coefficient of variation; VT: tidal volume; TO: lung turnovers, calculated as cumulative expired volume/FRC; DCDI: diffusion convection-interaction-dependent inhomogeneity; Sacin: DCDI contribution to first breath SnIII. CDI: convection-dependent inhomogeneity; Scond: rate of increase of SnIII from 1.5-6 TOs, also CDI contribution to SnIII; VC: vital capacity. 


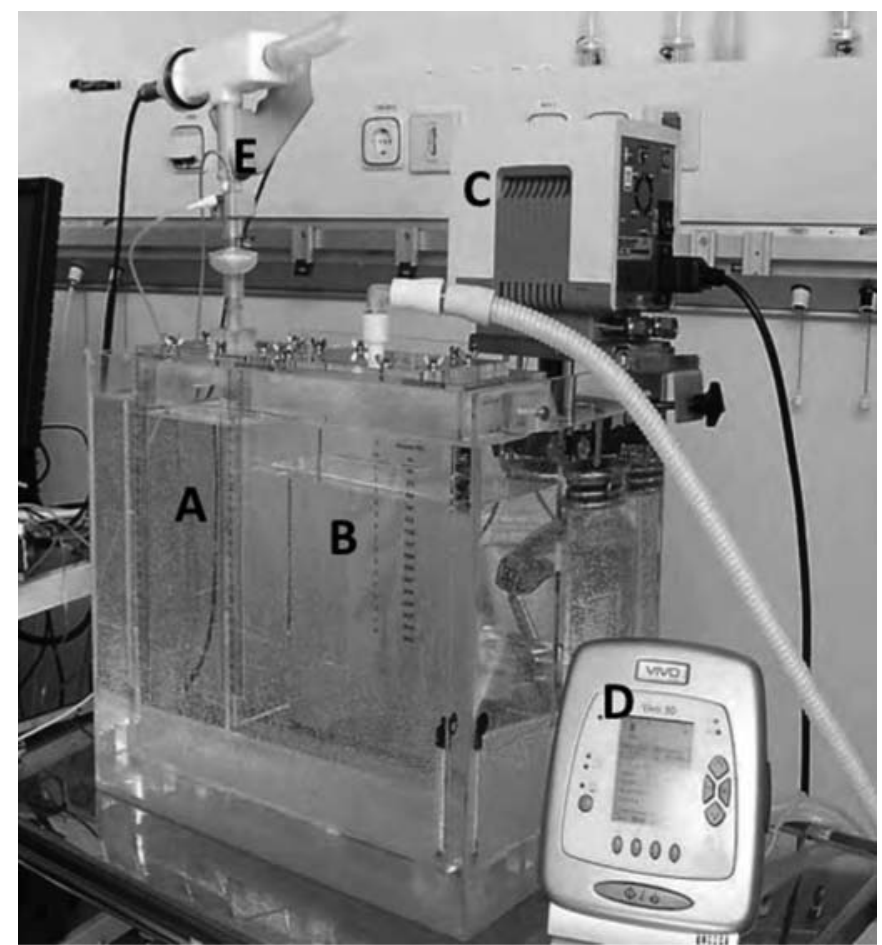

FIGURE 4. Recommended lung model for functional residual capacity (FRC) validation incorporating body temperature, ambient pressure, saturated with water vapour (BTPS) conditions and mimicking in vivo clinical testing conditions. The lung model consists of two separate chambers, an inner and an outer chamber. The inner chamber is partially divided (communicating at its inferior aspect) into two compartments: the lung compartment (A) and the ventilated compartment (B). FRC volume is generated by filling the inner chamber with distilled water to a measured height and calculated from known geometric dimensions. Water in the outer chamber is heated $(C)$ such that inner chamber water temperature reaches $37^{\circ} \mathrm{C}$, and a portable ventilator $(D)$ is connected to the ventilated compartment of the inner chamber and transmitted hydraulic pressure generates the lung chamber breathing pattern: chosen to simulate physiological tidal volume (VT)/FRC, VT and respiratory rates likely to be encountered during intended clinical testing [35]. For example, whilst $V_{T}$ remains similar $\left(8 \mathrm{~mL} \cdot \mathrm{kg}^{-1}\right)$ across age ranges, FRC changes from $\sim 20 \mathrm{~mL} \cdot \mathrm{kg}^{-1}$ in infants [37] to $40 \mathrm{~mL} \cdot \mathrm{kg}^{-1}$ in adults [38]. Multiple-breath washout equipment can be attached to the outlet of the lung compartment $(E)$ during validation tests.

Measured FRC can be corrected to represent different points in the airstream: FRC at the airway opening is calculated as FRC measured at the gas sampling point, FRCgs, minus pre-gas sampling point $V D$. FRC used in ventilation inhomogeneity index calculations must correspond to a common airstream measurement point (see section E6.2 in the online supplementary material).

Calculated FRC may continue to increase through the washout, particularly in subjects with airway disease and in $\mathrm{N}_{2}$-based MBW (see section entitled Impact of inert gas choice), yet studies rarely disclose when FRC measurement is determined. FRC end analysis threshold should correspond to the end-test threshold used for ventilation inhomogeneity indices (e.g. 1/40 of starting end-tidal concentration for lung clearance Index (LCI). The effect of variation in FRC end-point on other FRCderived indices may be significant. Methodology for reported FRC values should be clearly described.

\begin{tabular}{|c|c|}
\hline TABLE 6 & $\begin{array}{l}\text { that lead to variation in measured indices } \\
\text { n centres and recording systems }\end{array}$ \\
\hline Factor & Examples \\
\hline \multirow[t]{4}{*}{ Equipment related } & Analyser rise time \\
\hline & Analyser linearity \\
\hline & Flow measurement linearity \\
\hline & $\begin{array}{l}\text { Size of equipment related deadspace volume, } \\
\text { including distance between gas and flow } \\
\text { measurement points }\end{array}$ \\
\hline \multirow[t]{3}{*}{ Procedure related } & Inert gas used (and concentration) \\
\hline & Breathing stability \\
\hline & Age of subjects tested \\
\hline \multirow[t]{6}{*}{ Analysis related } & Algorithms used for calculation of indices \\
\hline & BTPS correction applied \\
\hline & $\begin{array}{l}\text { Corrections applied for equipment-related } \\
\text { deadspace }\end{array}$ \\
\hline & Drift correction algorithms \\
\hline & $\begin{array}{l}\text { Synchronisation of flow and gas concentration } \\
\text { signals }\end{array}$ \\
\hline & $\begin{array}{l}\text { Acceptability criteria applied (e.g. FRC values } \\
\text { within } 10 \% \text { ) }\end{array}$ \\
\hline
\end{tabular}

BTPS: body temperature, ambient pressure, saturated with water; FRC: functional residual capacity.

\section{Measures of ventilation distribution inhomogeneity}

A large number of ventilation distribution indices can be derived from information contained within SBW or MBW [21, 54,55] (see section E6.1 in the online supplementary material): 1) SBW SIII, reflecting combined CDI and DCDI contributions, unless simultaneously performed with marker gases of widely different MM. 2) MBW global ventilation inhomogeneity indices, reflecting efficiency of marker gas clearance. 3) MBW SnIII analysis, distinguishing CDI and DCDI mechanisms. 4) Airway closure and trapped gas volume (VTG) assessment from SBW and MBW, respectively.

Depending on the pathology under study, relationships between MBW-derived indices (e.g. Sacin, Scond and LCI) may help identify the type of structural changes generating increased ventilation distribution inhomogeneity [56].

\section{Global measures}

LCI is the most commonly reported MBW index in current paediatric literature, and defined as the number of FRC lung turnovers (TO; calculated as $\mathrm{CEV} / \mathrm{FRC}$ ) required to reduce alveolar tracer-gas concentration to a given fraction of its starting concentration, historically $1 / 40$ (2.5\%) [57]. Alveolar tracer-gas concentration has been estimated in various ways. In paediatric studies Cet is widely used, despite potential variability in end-tidal point. Identification of end-test threshold for LCI has not been systematically validated, but we recommend using the first of three consecutive breaths with a Cet $<1 / 40$ to avoid premature test termination with small breaths. LCI is calculated as the ratio of cumulative expired volume (CEV) to FRC, with CEV defined as the sum of all expiratory $V \mathrm{~T}$ over the washout including this first postthreshold. This introduces a small bias (overestimation); 
however, the value of interpolated or more complicated curve fit methods to determine exact threshold crossing values is unclear. Alternate methods used should be explicitly stated.

Ideally indices should be assessed at airway opening without external VD. However, this is, not feasible and $V$ T should be corrected for equipment $V \mathrm{D}$ as appropriate (see section E6.2 in the online supplementary material). Post-gas sampling point $V \mathrm{D}$ can be reliably estimated from water displacement; however, pre-gas sampling point $V \mathrm{D}$ determination may be challenging, due to streaming within the facemask or filter [58]. Applied pre- and post-gas sampling point corrections should be clearly described. Where VD correction is implemented, it is advised that both corrected and uncorrected LCI values are reported.

In clinical and modelling studies indices, such as LCI, have small but significant relationships to underlying respiratory patterns $(V \mathrm{~T}, V \mathrm{D}$ and FRC) particularly under disease conditions $[54,59,60]$. Effects of variation in respiratory rate and $V \mathrm{~T}$ can be minimised using moment analysis (see section E6.4 in the online supplementary material). This describes the degree of skewness of the washout curve to the right, as mean dilution numbers (MDN) or moment ratios [61]. VD-independent assessment is feasible by correcting CEV for airways $V \mathrm{D}$ $(V \mathrm{D}, \mathrm{aw})$ and using cumulative expired alveolar volume in calculations (CEValv; e.g. alveolar MDN [59] and alveolar LCI [62]). VD,aw, measured using Fowler or Langley methods (see section E4.2 in the online supplementary material) [63, 64], should be based on $\mathrm{CO}_{2} V \mathrm{D}$,aw, or the first few washout breaths of inert gas $V \mathrm{D}$,aw, as the latter increases during MBW [25] due to early washout of very well ventilated lung regions with short pathways to the airway opening. However, moment ratio truncation to facilitate between-subject comparison (e.g. to 8 TO [65]), may detrimentally affect sensitivity [66], and feasibility. Healthy subjects may also require longer washout periods to reach these higher turnover values, and accurate measurement may be compromised by limited signal resolution and high relative noise at the low gas concentrations encountered.

\section{Normalised SnIII analysis}

MBW SnIII analysis has a theoretical [67], experimental [68], and lung modelling basis [69-72] from morphometric data in healthy adults [22], to distinguish ventilation inhomogeneity arising from DCDI and CDI mechanisms, expressed as the clinical indices Sacin and Scond, respectively [72] (fig. 5). For Sacin and Scond determination, SIII and gas concentrations must be accurately determined down to breaths with very low concentrations (see section E6.6 in the online supplementary material) and may not be feasible for all washout systems.

SIII is dependent on many factors, both linear and non-linear, at least in healthy adult lungs: pre-inspiratory lung volume, inspired and expired volumes, and flow [1, 20, 42, 73-78]. Consequently, these factors should ideally be kept similar between subjects to maintain diffusion-convection front location, and allow changes in indices to be linked to changes in corresponding lung structures. Breath holds at end-inspiration flatten SIII and should be minimised [30,63]. The beating heart generates flow pulses within airways [79] causing cardiogenic gas mixing. Cardiogenic oscillations superimposed onto SIII

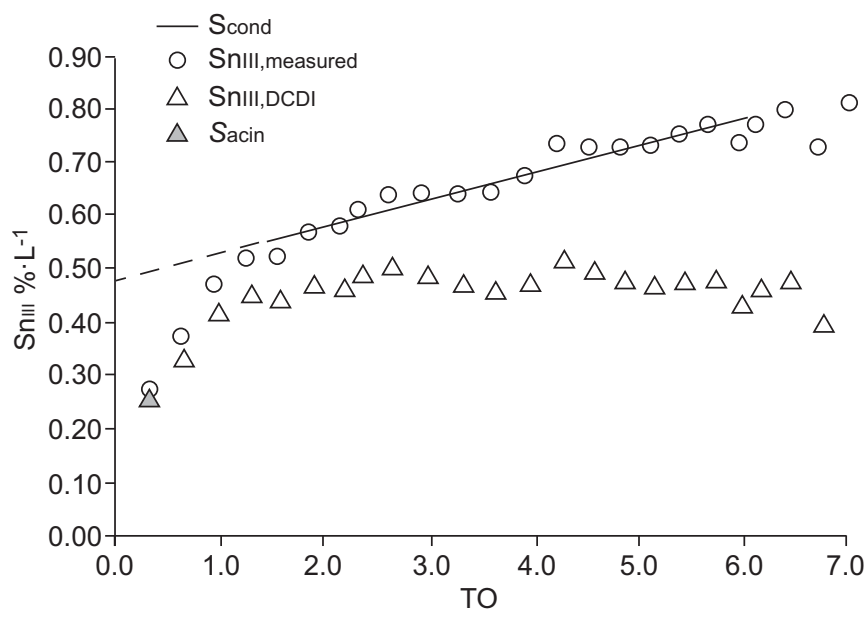

FIGURE 5. Normalised phase III slope (SnIII) analysis. Multiple-breath washout recording illustrating derived phase III slope parameters. Measured concentration SnIII, (SnIII,measured) for each breath is plotted against its corresponding lung turnover, calculated as cumulative expired volume/functional residual capacity (TO), value. Progressive SnIII values increase throughout the TO range considered. If this does not occur, the quality of the recording should be closely examined. The index of convection-dependent inhomogeneity (CDI; Scond), is calculated as the increase in measured SnIII per unit TO between $\sim 1.5-6.0$ TO per unit TO. For explanation purposes the diffusion convection-interaction-dependent inhomogeneity (DCDI) contribution to the SnIII for each breath is also plotted (SnIII,DCDI) This is calculated by subtracting the CDI contribution to SnIII for each breath from the SnIII,measured for each breath. In other words, for each breath, SnIII,measured value equals SnIII,DCDI value minus CDI contribution. Sacin is defined as the DCDI contribution to the first breath SnIII,DCDI. The complete contribution of the DCDI mechanism reaches a plateau beyond TO 1.5 and is equivalent to the intercept of the Scond regression line. These indices rely on the fact that DCDI generates a horizontal asymptote and CDI does not and are, therefore, only valid in cases where SnIII does not show a horizontal asymptote.

add to signal noise. Automated SnIII calculation algorithms exist [80], but subjective observation is still necessary to review estimated slope accuracy.

\section{Trapped gas volume}

Airway closure occurs in lung units approaching regional RV [81], but may also occur at higher regional lung volumes in infants, older adults [82], obese subjects [83], and in the presence of peripheral airway obstruction. It may be a prominent phenomenon in airway disease. If present, the VTG can be measured during MBW by including five inspiratory capacity breaths after conventional end-test threshold is reached and measuring the volume of lung recruited (see section E6.3 in the online supplementary material). VTG measurement with both resident and exogenous MBW has been established for infants and children [84, 85]. Importantly, this method estimates only the gas volumes recruitable during these large breaths.

\section{Closing volume and closing capacity}

Closing volume (CV) and closing capacity require accurate determination of SBW phase III to phase IV transition (fig. 1). $\mathrm{CV}$ reflects airway closure occurring preferentially in dependent lung regions and peripheral airway obstruction [81, 86, 87]. Relative merits of these indices have been reviewed elsewhere [88]. Although feasible in adults [89], paediatric 
utility of CV is limited [90]. Automated identification of phase IV is feasible [91].

\section{IMPACT OF INERT GAS CHOICE}

Derived indices may differ depending on the gas used for a number of reasons. Gas diffusion rate is inversely proportional to the square root of the $\mathrm{MM}$, but convective distribution is unaffected. Consequently, diffusion-convection front location is more proximal for lighter gases versus heavier gases (e.g. He versus $\mathrm{SF}_{6} \mathrm{MM}$ is 4 versus $146 \mathrm{~g} \cdot \mathrm{mol}^{-1}$, respectively). Greater series $V \mathrm{D}$ for $\mathrm{SF}_{6}$, compared to He, generates higher $\mathrm{LCI}$ values, irrespective of ventilation distribution itself. In healthy lungs $\mathrm{SF}_{6}$ SIII are greater than He SIII, but may reverse in lung pathology [92-94]. In addition, rate of diffusive equilibration in enlarged peripheral air spaces (e.g. emphysema) may differ depending on gas choice generating differential SIII increase. Homogeneity of gas distribution present at the start of washout may differ depending on whether naturally resident or exogenous gas is used. Measurable differences may be informative. In simultaneous $\mathrm{He}$ and $\mathrm{SF}_{6}$ measurements, disease processes distal to the acinar entrance generate greater abnormality in $\mathrm{SF}_{6}$ indices, whereas disease processes proximal to the acinar entrance but in the zone of the convection-diffusion front will affect He indices preferentially. However, if disease processes affect $\mathrm{SF}_{6}$ and $\mathrm{He} \mathrm{SIII}$ to a similar extent, no relative SIII difference occurs [95].

Advantages of $\mathrm{N}_{2}$ washout include widespread availability and affordability of $100 \% \mathrm{O}_{2}$, and avoidance of patient connection to equipment during wash-in periods between tests minimising patient discomfort. $\mathrm{N}_{2}$ is resident in all lung units including very slowly ventilated lung compartments and may offer improved sensitivity to detect abnormality, compared to other inert gases, which may not equilibrate fully within these regions during wash-in. However, disadvantages also exist. Thresholds at which factors such as age, sleep state and sedation interact with $100 \% \mathrm{O}_{2}$ to affect breathing pattern remain unclear. $\mathrm{N}_{2}$ is not truly inert and tissue $\mathrm{N}_{2}$, present due to high atmospheric $\mathrm{N}_{2}$ partial pressure, diffuses from blood into alveoli along concentration gradients. This diffusion is greatest in well-ventilated lung regions washed out during initial portions of the test, and contribute to exhaled $\mathrm{N}_{2}$ later in the washout, potentially introducing greater error in longer tests (e.g. FRC overestimation). Estimation and correction of tissue $\mathrm{N}_{2}$ contribution is difficult due to limited available data to base correction [96], and adjustment for tissue $\mathrm{N}_{2}$ is not currently recommended [97].

Whilst different inert gas concentrations used in the literature are safe (e.g. $4 \% \mathrm{SF}_{6}$ and $\left.4 \% \mathrm{He}\right)$, additional factors influence inert gas selection. $\mathrm{SF}_{6}$ may have adverse health effects at higher concentrations [98] and significant greenhouse potential [99]. Feasibility of scavenging following testing is unclear. $\mathrm{SF}_{6}$ is not universally approved for testing (e.g. USA and France). Low density of He renders it more susceptible to leaks during testing, which may aid leak detection. Cost of exogenous gas is increasing in many countries, partly due to increasing logistical requirements when used as a medical gas.

\section{ACCEPTABILITY CRITERIA FOR TESTING}

Quality control during testing is critical and extends beyond equipment performance and software feedback to also include close observation by the operator of the subject's behaviour during testing and how this affects the data obtained. Adequate operator training and appreciation of all factors influencing test results is essential. Recommended acceptability criteria for MBW and SBW are summarised in tables 7 and 8.

\section{Multiple-breath washout}

Primary index of interest may differ between paediatric and adult testing (e.g. LCI and SnIII indices in the current literature, respectively) influencing test termination criteria and acceptability. Recommendations contained within this document attempt to provide a unified approach.

\section{Breathing pattern}

Measured FRC reflects lung volume at which washout is commenced (i.e. end-expiratory level). Stability of resting lung volumes before and throughout washout is critical $[46,48]$. In infants, intrinsic FRC resetting during critical periods, visible as sighs, should prompt test exclusion. In general, large inspiratory breaths during washout may mobilise trapped gas and small inspiratory or expiratory breaths may result in steeper SIII. End-tidal volumes below FRC may result in steeper SIII and occurrence of phase IV, especially in obstructive lung disease. For SnIII analysis, first breath quality (in particular adherence to target inhalation and exhalation volume) is critical for accurate Sacin.

Relaxed tidal breathing has historically been used for global MBW derived indices. Studies introducing adult SnIII analysis used a strict $1 \mathrm{~L} \mathrm{VT}$ breathing regimen [101], chosen as a compromise between 1) maintaining physiological breathing conditions, 2) obtaining sufficient phase III to compute its slope, and 3) having sufficient SnIII data points for statistically valid regression from $\sim$ TO 1.5-6.0 [101]. This strict protocol is not feasible in all ages, or in more advanced obstructive disease. In addition, due to marked variations in lung size, $1 \mathrm{~L}$ may greatly exceed normal $V_{\mathrm{T}}$ and not be appropriate. In an attempt to implement SnIII analysis in younger ages during regular breathing (typically aged $\leqslant 16 \mathrm{yrs}$ ), the following criterion for breath acceptability, based on a similar principle, is proposed: each breath must have sufficient phase III to compute SIII (at least $50 \%$ of $V \mathrm{~T}$ ). For tests fulfilling this criterion, volume compensation is then performed on SnIII: SnIII is multiplied by FRC (to correct for differences in lung size) and then by VT/FRC (to account for variations in SIII due to changes in breathing pattern). This net multiplication of SnIII by $V$ T (in $\mathrm{L}$ ) facilitates comparison among subjects of differing lung sizes, yet needs to be critically interpreted in any particular study setting (see section E6.5 in the online supplementary material). Where implemented, we recommend that both corrected and uncorrected Sacin and Scond values are reported, such that posteriori analyses are possible, if and when this or other correction methods are validated. Insufficient SIII for accurate estimation limits feasibility in infants [105].

Visual breathing pattern feedback may be useful to guide older adolescents and adults [9] but is problematic in younger subjects, for whom distraction with videos is recommended [6]. Measurements in infants should be performed during quiet nonrapid eye-movement sleep, with or without the use of sedation. No comparative study exists showing the potential effect of sedation on washout indices. 


\section{TABLE 7 Multiple-breath washout (MBW) measurement acceptability criteria}

\section{Testing position}

Infants: supine position with head in midline and in sniffing position to optimise upper airway patency

Preschool and above: seated position, with head in midline

\section{Interface}

Infants and preschoolers: suitable facemask with adequate therapeutic putty volume to ensure adequate facemask seal and reduction of the pre-gas sampling point VD without obstructing the airway opening

Nasal mask measurements are feasible during periods of preferential nasal breathing [100] but require further study

Box shaped flow-volume loops may indicate an upper airway obstruction or external obstruction of the airway opening by therapeutic putty

Older subjects: nose clip and maintain tight mouthpiece seal

\section{Three technically acceptable MBW runs should be performed, with acceptability defined by the following criteria}

Wash-in phase (or pre-washout phase for $\mathrm{N}_{2} \mathrm{MBW}$ )

Stable $V_{T}$ and end-expiratory lung volume over the preceding $30 \mathrm{~s}$

Deviation in end-expiratory lung volume at start of test within 10\% of mean VT of preceding five breaths

An irregular small volume breath immediately prior to starting the washout may also lead to error in end tidal estimate of starting alveolar concentration

Equilibration of exogenous wash-in gas within the breath cycle (i.e. inspiratory versus expiratory end tidal concentration)

Variability $<1 \%$ relative to mean inspired concentration (i.e. $<0.04 \%$ if the inspired concentration is $4 \%$ )

Adequate starting end-tidal inert gas concentration, stable over $30 \mathrm{~s}$ (i.e. equal to inspired gas concentration)

Washout phase

Regular breathing pattern

Sufficient breath size for adequate phase III slope identification (if SnIII analysis being performed)

Breathing protocols of $1 \mathrm{~L} V$ T are recommended in older adolescents (e.g. $>16 \mathrm{yrs}$ ) and adults but may not be feasible in all age groups (e.g. VT 1.0-1.3 L) [101-103]

No evidence of significant trapped gas release with larger breaths; release of trapped gas

Invalidates SnIII analysis and increases measured LCl

May be difficult to avoid in advanced CF lung disease

No coughing

Specific to infants during critical periods of the wash-in/washout

No evidence of apnoeas (may significantly decrease FRC)

No evidence of sighs (may significantly elevate FRC)

Critical period defined as the 10 breaths prior to achieving equilibration or during the first 10 breaths of the washout

Criteria for test termination

At least three consecutive breaths with end tidal inert gas concentration values below $1 / 40$ of starting inert gas concentration

If SnIII analysis alone, then at least 6 TO must be included

If moment analysis is being performed then at least 6 TO should be included, as data collected at 8 TO in normal subjects are likely to be compromised by poor gas signal quality

No evidence of leak occurring during the test

Resident inert gas (e.g. $\mathrm{N}_{2}$ ) - leak indicated by the following during the washout phase

Sudden spike in $\mathrm{N}_{2}$ concentration during inspiration (consistent with post-gas sampling point inspiratory air leak)

Premature rise in $\mathrm{N}_{2}$ signal early in expirogram of following breath, where $\mathrm{N}_{2}$ concentrations should be zero in the initial absolute dead space portion (consistent with pre-gas sampling point inspiratory air leak)

VD,aw decrease

Sudden step changes of the volume trace

Step-up of $\mathrm{N}_{2}$ concentration plotted versus TO

Exogenous inert gas: leak indicated by

Failure of equilibration between inspiratory and expiratory inert gas concentrations during wash-in (consistent with pre- or post-gas sampling point leak)

Sudden drop in inspiratory inert gas concentration during wash-in (consistent with post-gas sampling point leak)

VD,aw increase during washout

Sufficient interval between runs when using resident inert gases to allow inert gas concentration to return to baseline values

Twice the washout time is a conservative recommendation. If a shorter interval is used, then the operator must demonstrate that alveolar concentrations has been resituated [104]

This period may be lengthy in advanced obstructive disease

Inadequate duration may significantly decrease measured FRC

The following should trigger further investigation for artefact but are not a reason to exclude tests alone

Marked volume drift during testing or sudden changes in volume (without other evidence of leak)

$\mathrm{FRC}$ or $\mathrm{LCl}$ variability $>10 \%$, measured as the difference between maximum and minimum values

Tests where FRC differs by $\mathbf{>} 25 \%$ from the median FRC value across the three tests should be automatically rejected

Test equipment and performance must adhere to infection control guidelines

Use of bacterial filters may significantly increase VD and preclude the use of certain systems in younger age groups

$\mathrm{N}_{2}$ : nitrogen gas; VD: deadspace volume; VT: tidal volume; SnIII: normalised phase III slope; LCl: lung clearance index; CF: cystic fibrosis; FRC: functional residual capacity; TO: lung turnovers, calculated as cumulative expired volume/FRC; $V D$, aw: deadspace volume of the conducting airways 


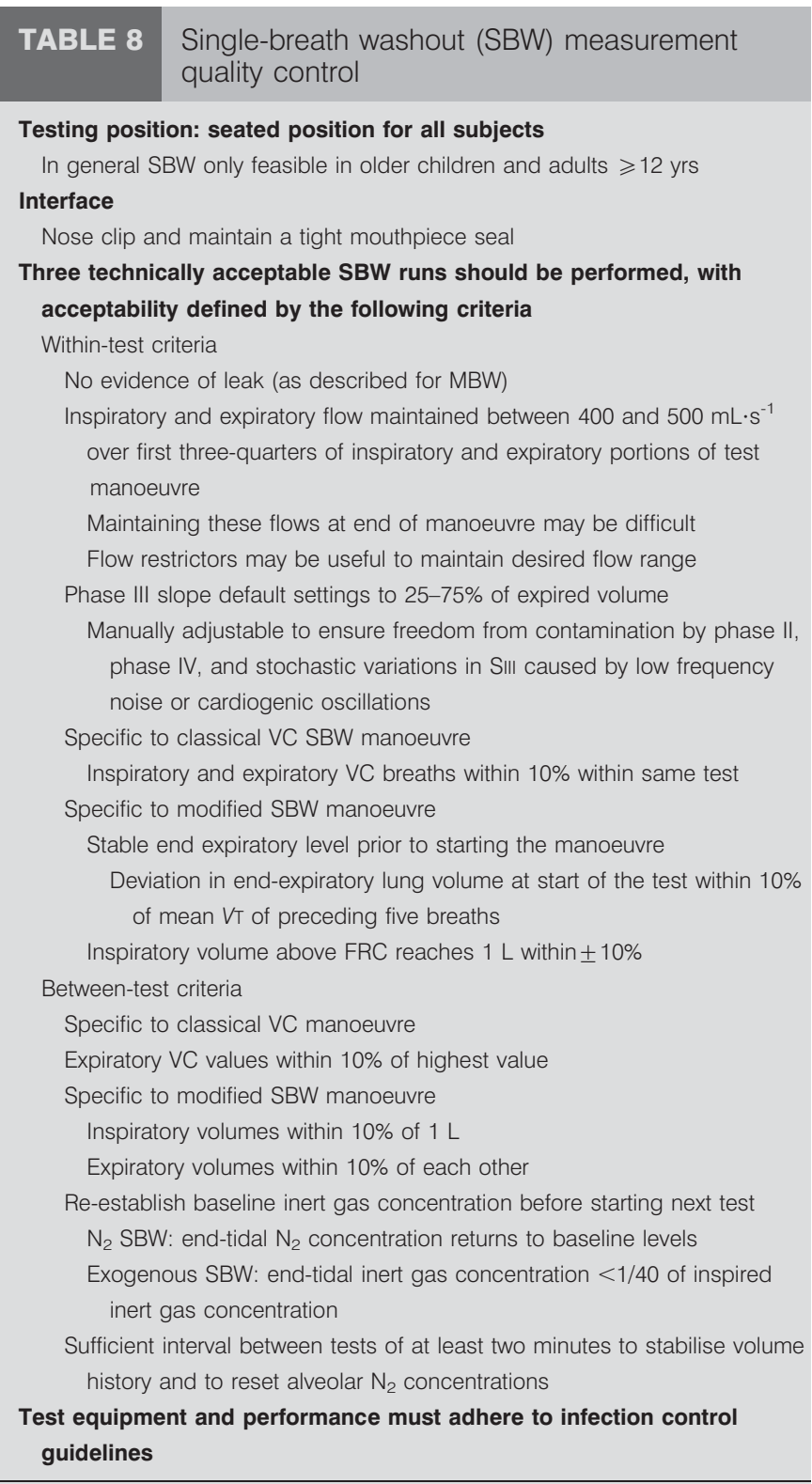

MBW: multiple-breath washout; SIII: phase III slope; VC: vital capacity; VT: tidal volume; FRC: functional residual capacity; $\mathrm{N}_{2}$ : nitrogen gas.

\section{Test termination}

MBW test termination after alveolar concentration (usually Cet) falls below $1 / 40$ of starting concentration for three consecutive breaths allows standard LCI to be calculated. For standard SnIII analysis and moment ratios MBW should pass beyond 6 and $8 \mathrm{TO}$, respectively [65].

\section{FRC repeatability}

Previously recommended within-session FRC repeatability criteria (within 10\%, [106, 107]) have poor feasibility in paediatric testing [108], and may lengthen total testing time significantly. Repeatability within $10 \%$ should be viewed as encouraging. Tests should be carefully examined for technical issues if this is not met. Automatic exclusion of tests should occur if FRC differs by $>25 \%$ from median FRC over three tests.
In older children, FRC increases by $>20 \%$ moving supine to sitting [109], but effects of transition from testing supine infants to seated preschoolers is unclear. Postural effects on ventilation distribution may also depend on severity and topographical location of airways disease. Consideration of these factors should also occur when comparing upright ventilation distribution tests to supine imaging studies.

\section{Single-breath washout}

The need to maintain inspiratory and expiratory flows strictly between $400-500 \mathrm{~mL} \cdot \mathrm{s}^{-1}$ and achieve reproducible VC manoeuvres currently limits feasibility to adults and children $>12$ yrs [18]. SIII volume compensation, using a similar approach to MBW, in this case by multiplying SIII by VC, is feasible but not formally validated. It is unclear how much variation in historical predicted SIII values [18] is due to physiological intrinsic or technical factors.

\section{FUTURE WORK AND CONCLUSIONS}

Important questions remaining unanswered for commercial and research washout systems, SBW and MBW test procedure and subsequent analysis are summarised in table 9. Challenges arise when interpreting washout tests in infants and children where relationships between $V \mathrm{D} / V \mathrm{~T}$ and $V \mathrm{~T} / \mathrm{FRC}$ and calculated indices must be considered. This is particularly relevant when undertaking studies of early lung disease or treatment effects to ensure that reported differences don't reflect alterations in respiratory patterns alone. Longitudinal data for ventilation inhomogeneity indices during normal lung development with age are needed. Influence of sex and ethnic background is unclear.

Anatomical distinction between ventilation inhomogeneity represented by Scond and Sacin relies on diffusion-convection front location, which has been simulated in an adult lung using available lung structure and airway dimensions. Extending applicability of such indices into childhood and disease processes requires further simulation of the diffusion-convection front based on realistic anatomical data. Beyond post mortem data, anatomical and functional data obtained using modern computed tomography scanning techniques or hyperpolarised noble gas magnetic resonance imaging studies may provide this. Simulation studies in realistic lung models could also be used to validate $V \mathrm{~T}$ correction of SnIII to compare ventilation inhomogeneity between varying age groups with varying $V \mathrm{D}, V \mathrm{~T}$, and FRC. Until formal validation, studies incorporating SnIII analysis should ideally include matched healthy control data for comparison and report both uncorrected and corrected values. Formal objective quality control thresholds for test acceptance and breath exclusion are also required. Shortening test duration whilst maintaining sensitivity and specificity will enhance feasibility and incorporation into routine clinical testing. Efforts to investigate ways to achieve this are already underway [108, 112].

Inert gas washout provides unique physiological information, which at the very least forms an important complement to current methods in the adult lung function laboratory, while offering improved feasibility and sensitivity compared to spirometry in younger children. A number of important challenges lie ahead for integration into routine clinical care. Standardisation of procedures and development of robust 
TABLE 9 Important areas of interest for future studies

Area of interest
Equipment validation
Synchronisation of gas and flow signals
BTPS correction
Equipment VD estimation
Gas analyser properties
Sample flow (Sidestream gas analysers
Tissue $\mathrm{N}_{2}$
$\mathrm{~N}_{2}$-based MBW
Use of sedation in infants
Measures of global ventilation
inhomogeneity
Importance of FRC repeatability
Normative data
MBW SnIII analysis

Commercial devices

\section{Questions and needs}

Feasible validation methods for end-tidal inert gas concentration and phase III slope measurement Optimal synchronisation method, protocol for measurement, and the thresholds for acceptable synchronisation error remain unclear

Optimal BTPS correction. Is dynamic BTPS correction required during testing? How are changes in temperature and relative humidity most accurately measured during inspiration and expiration?

Accurate estimation of effective external VD. Streaming may occur with equipment-related VD. Therefore water displacement measurement of $V_{D}$ may overestimate influence of $V_{D}$,ext on breathing pattern. This includes facemasks and in-line bacterial filters

Acceptable maximum response time for different age groups and breathing patterns?

Degree of error introduced by sample flow: What is an acceptable sample flow? Given its age-dependence, should it be considered as a \% of $V T$ ? What is the most appropriate method to correct flow and marker gas volume for sample flow?

Effective correction for effect of tissue nitrogen diffusing into alveoli during washout. What is the error introduced into subsequent indices (FRC, $\mathrm{LCl}$ and SnIII analysis)?

At what age does $100 \% \mathrm{O}_{2}$ no longer have a detrimental effect on breathing pattern?

Effect of sedation on ability of infants to actively maintain FRC or effect on breathing pattern? This has been speculated upon but remains unproven $[110,111]$

Can test duration be shortened whilst preserving acceptable sensitivity?

Flexibility of current MBW end-test thresholds (e.g. evaluation of $1 / 20$ for $\mathrm{LCl}$ and 6 TO for moment ratios) How many tests are needed to give an accurate estimate? [108]

Can wash-in data also be utilised to calculate indices?

Utility of interpolation or curve fitting methods to determine exact end-of-test for $\mathrm{LCl}$

Validation of pre- and post-gas sampling point VD corrections

Validity of paediatric correction of SnIII by VT to account for differences in tidal VT and breathing pattern Most appropriate inert gas reference concentration for normalisation of SIII

Formal objective criteria for exclusion of outlying SnIII values

Can accurate estimates be obtained from two tests?

Influence of geometric choice within the airstream and the time point chosen for FRC determination during the washout on FRC, CEV and TO on subsequently reported ventilation inhomogeneity indices FRC repeatability recommendations here are based on consensus and further research is needed to define these in future studies; the impact of FRC variability on SnIII indices is unclear

Validity of paediatric correction of SIII by expiratory VC to account for differences in lung size Normative data needs to be collected for indices across different age, sex and ethnic groups.

Standardisation of procedures is essential if results are to be comparable across centres and between devices. Differences in results obtained among gases with different molecular masses are expected;

formal comparisons are lacking

Development of robust accurate commercial devices which can be used across wide age ranges

BTPS: body temperature, ambient pressure, saturated with water; VD: deadspace volume; $\mathrm{N}_{2}$ : nitrogen gas; MBW: multiple-breath washout; SnIII: normalised phase III slope; FRC: functional residual capacity; CEV: cumulative expired volume; TO: lung turnovers, calculated as CEV/FRC; SBW: single-breath washout; SIII: phase III slope; VD,ext: external equipment deadspace volume; VT: tidal volume; LCl: lung clearance index; VC: vital capacity.

appropriately validated affordable commercial equipment is essential. This will only be achievable if manufacturers work in collaboration with researchers, as we seek to address the important issues and questions that remain unanswered. This standardisation document provides the basis for this future work.

\section{STATEMENT OF INTEREST}

Statement of interest for P.D. Robinson, M. Gappa, G.G. King, J.J. Pillow, and F. Ratjen can be found at www.erj.ersjournals.com/site/ misc/statements.xhtml

\section{ACKNOWLEDGEMENTS}

This consensus statement has been endorsed by the European Respiratory Society and the American Thoracic Society.
The author affiliations are as follows. P.D. Robinson: Dept of Respiratory Medicine, The Children's Hospital at Westmead, and Airway Physiology and Imaging, Woolcock Institute of Medical Research, Sydney, Australia, and UCL Institute of Child Health, Portex Respiratory Unit, London, UK; P. Latzin: Division of Respiratory Medicine, Dept of Paediatrics, Inselspital and University of Bern, Bern, Switzerland; S. Verbanck: Respiratory Division, University Hospital, Brussels, Belgium; G.L. Hall: Telethon Institute for Child Health Research, Centre for Child Health Research, University of Western Australia, and Respiratory Medicine, Princess Margaret Hospital for Children, Perth, Australia, and Division of Respiratory Medicine, Physiology and Experimental Medicine, Hospital for Sick Children, Toronto, ON, Canada; A. Horsley: Manchester Adult Cystic Fibrosis Centre, University Hospitals South Manchester, Wythenshawe Hospital, Manchester, UK; M. Gappa: 
Children's Hospital and Research Institute for the Prevention of Allergies and Respiratory Diseases in Children, Marien-Hospital, Wesel, Germany; C. Thamrin: Airway Physiology and Imaging, Woolcock Institute of Medical Research, Sydney, Australia, and Division of Respiratory Medicine, Dept of Paediatrics, Inselspital, and University of Bern, Bern, Switzerland; H.G.M. Arets: Dept of Paediatric Pulmonology, Wilhelmina Children's Hospital, University Medical Center Utrecht, Utrecht, The Netherlands; P. Aurora: UCL Institute of Child Health, Portex Respiratory Unit, and Respiratory Medicine, Great Ormond Street Hospital for Children NHS Trust, London, UK; S. Fuchs: Children's Hospital and Research Institute for the Prevention of Allergies and Respiratory Diseases in Children, Marien-Hospital, Wesel, Germany; G.G. King: Airway Physiology and Imaging, Woolcock Institute of Medical Research, and Dept of Respiratory Medicine, Royal North Shore Hospital, Sydney, Australia; S. Lum: UCL Institute of Child Health, Portex Respiratory Unit, London, UK; K. Macleod: Dept of Child Life and Health, Royal Hospital for Sick Children, University of Edinburgh, Edinburgh, UK; M. Paiva: Respiratory Division, University Hospital Erasme, Université Libre de Bruxelles, Brussels, Belgium; J.J. Pillow: Centre for Neonatal Research and Education, School of Paediatrics and Child Health and School of Women's and Infants' Health, University of Western Australia, and King Edward and Princess Margaret Hospitals, Perth, Australia; S. Ranganathan: Dept of Respiratory Medicine, Royal Children's Hospital, and Murdoch Children's Research Institute, Melbourne, Australia; F. Ratjen: Division of Respiratory Medicine, Dept of Paediatrics, The Hospital for Sick Children, Toronto, ON, Canada; F. Singer: Division of Respiratory Medicine, Dept of Paediatrics, Inselspital and University of Bern, Bern, Switzerland; S. Sonnappa: UCL Institute of Child Health, Portex Respiratory Unit, and Respiratory Medicine, Great Ormond Street Hospital for Children NHS Trust, London, UK; J. Stocks: UCL Institute of Child Health, Portex Respiratory Unit, London, UK; P. Subbarao: Division of Respiratory Medicine, Dept of Paediatrics, The Hospital for Sick Children, Toronto, ON, Canada; B.R. Thompson: Physiology Service, Dept of Allergy, Immunology and Respiratory Medicine, The Alfred Hospital, Melbourne, Australia; P.M. Gustafsson: Dept of Paediatrics, Central Hospital, Skövde, and Sahlgrenska Academy, University of Gothenburg, Gothenburg Sweden.

Other members of the working group who contributed early on in the production of this work were H.A.W.M. Tiddens, (Rotterdam, the Netherlands), S.M. Schulzke (Basel, Switzerland), A. Schibler (Brisbane, Australia) and R.S. Tepper (Indianapolis, IN, USA).

\section{REFERENCES}

1 Fowler WS. Lung function studies; uneven pulmonary ventilation in normal subjects and in patients with pulmonary disease. J Appl Physiol 1949; 2: 283-299.

2 Robertson JS, Siri WE, Jones HB. Lung ventilation patterns determined by analysis of nitrogen elimination rates; use of mass spectrometer as a continuous gas analyzer. J Clin Invest 1950; 29: 577-590.

3 Venegas JG, Winkler T, Musch G, et al. Self-organized patchiness in asthma as a prelude to catastrophic shifts. Nature 2005; 434 : 777-782.

4 van Beek EJ, Hill C, Woodhouse N, et al. Assessment of lung disease in children with cystic fibrosis using hyperpolarized 3Helium MRI: comparison with Shwachman score, ChrispinNorman score and spirometry. Eur Radiol 2007; 17: 1018-1024.

5 Aurora P, Bush A, Gustafsson P, et al. Multiple-breath washout as a marker of lung disease in preschool children with cystic fibrosis. Am J Respir Crit Care Med 2005; 171: 249-256.

6 Aurora P, Gustafsson P, Bush A, et al. Multiple breath inert gas washout as a measure of ventilation distribution in children with cystic fibrosis. Thorax 2004; 59: 1068-1073.
7 Gustafsson PM, Aurora P, Lindblad A. Evaluation of ventilation maldistribution as an early indicator of lung disease in children with cystic fibrosis. Eur Respir J 2003; 22: 972-979.

8 Fuchs SI, Sturz J, Junge S, et al. A novel sidestream ultrasonic flow sensor for multiple breath washout in children. Pediatr Pulmonol 2008; 43: 731-738.

9 Horsley AR, Gustafsson PM, Macleod KA, et al. Lung clearance index is a sensitive, repeatable and practical measure of airways disease in adults with cystic fibrosis. Thorax 2008; 63: 135-140.

10 Aurora P, Stanojevic S, Wade A, et al. Lung clearance index at 4 years predicts subsequent lung function in children with cystic fibrosis. Am J Rep Crit Care Med 2011; 183: 752-758.

11 Kjellman B. Lung function in children with pneumonia. With special reference to distribution of inspired air and regional lung function. Scand J Respir Dis 1968; 49: 185-201.

12 Shao H, Sandberg K, Sjoqvist BA, et al. Moment analysis of multibreath nitrogen washout in healthy preterm infants. Pediatr Pulmonol 1998; 25: 52-58.

13 Hulskamp G, Lum S, Stocks J, et al. Association of prematurity, lung disease and body size with lung volume and ventilation inhomogeneity in unsedated neonates: a multicentre study. Thorax 2009; 64: 240-245.

14 Lum S, Gustafsson P, Ljungberg H, et al. Early detection of cystic fibrosis lung disease: multiple-breath washout versus raised volume tests. Thorax 2007; 62: 341-347.

15 Fuchs SI, Gappa M. Lung clearance index: clinical and research applications in children. Paediatr Respir Rev 2011; 12: 264-270.

16 Gangell CL, Hall GL, Stick SM, et al. Lung function testing in preschool-aged children with cystic fibrosis in the clinical setting. Pediatr Pulmonol 2010; 45: 419-433.

17 Horsley A. Lung clearance index in the assessment of airways disease. Respir Med 2009; 103: 793-799.

18 Robinson PD, Goldman MD, Gustafsson PM. Inert gas washout: theoretical background and clinical utility in respiratory disease. Respiration 2009; 78: 339-355.

19 Robinson PD, Latzin P, Gustafsson P. Multiple-breath washout. Eur Respir Monogr 2010; 47: 87-104.

20 Paiva M. Gas transport in the human lung. J Appl Physiol 1973; 35: 401-410.

21 Engel LA, Paiva M. Gas Mixing and Distribution in the Lung. Marcel Dekker, Inc., New York, 1985.

22 Haefeli-Bleuer B, Weibel ER. Morphometry of the human pulmonary acinus. Anat Rec 1988; 220: 401-414.

23 Engel LA. Gas mixing within the acinus of the lung. J Appl Physiol 1983; 54: 609-618.

24 Prisk GK, Lauzon AM, Verbanck S, et al. Anomalous behavior of helium and sulfur hexafluoride during single-breath tests in sustained microgravity. J Appl Physiol 1996; 80: 1126-1132.

25 Crawford $\mathrm{AB}$, Makowska M, Paiva M, et al. Convection- and diffusion-dependent ventilation maldistribution in normal subjects. J Appl Physiol 1985; 59: 838-846.

26 Comroe JH Jr, Fowler WS. Lung function studies. VI. Detection of uneven alveolar ventilation during a single breath of oxygen. Am J Med 1951; 10: 408-413.

27 Anthonisen NR, Robertson PC, Ross WR. Gravity-depende sequential emptying of lung regions. J Appl Physiol 1970; 28: 589-595.

28 Olson LE, Rodarte JR. Regional differences in expansion in excised dog lung lobes. J Appl Physiol 1984; 57: 1710-1714.

29 Wilson TA, Olson LE, Rodarte JR. Effect of variable parenchymal expansion on gas mixing. J Appl Physiol 1987; 62: 634-639.

30 Kjellmer I, Sandqvist L, Berglund E. Alveolar plateau of the single breath nitrogen elimination curve in normal subjects. J Appl Physiol 1959; 14: 105-108.

31 Van Muylem A, De Vuyst P, Yernault JC, et al. Inert gas singlebreath washout and structural alteration of respiratory bronchioles. Am Rev Respir Dis 1992; 146: 1167-1172. 
32 Frey U, Stocks J, Coates A, et al. Specifications for equipment used for infant pulmonary function testing. Eur Respir J 2000; 16: 731-740.

33 Bates JH, Schmalisch G, Filbrun D, et al. Tidal breath analysis for infant pulmonary function testing. Eur Respir J 2000; 16: 1180-1192.

34 Fuchs SI, Buess C, Gappa M. In vitro validation of nitrogen multiple breath washout using ultrasonic equipment. Eur Respir J 2011; 38: Suppl. 55, 355s.

35 Singer F, Houltz B, Latzin P, et al. A realistic validation study of a new nitrogen multiple-breath washout system. PLoS One 2012; 7: e36083.

36 Brunner JX, Wolff G, Cumming G, et al. Accurate measurement of N2 volumes during N2 washout requires dynamic adjustment of delay time. J Appl Physiol 1985; 59: 1008-1012.

37 Hulskamp G, Pillow JJ, Dinger J, et al. Lung function tests in neonates and infants with chronic lung disease of infancy: functional residual capacity. Pediatr Pulmono 2006; 41: 1-22.

38 Zeltner TB, Caduff JH, Gehr P, et al. The postnatal development and growth of the human lung. I. Morphometry. Respir Physiolo 1987; 67: 247-267.

39 Arieli R. Mass spectrometer for respiratory research. Respir Physiol Neurobiol 2010; 170: 183-184.

40 Amin R, Subbarao P, Jabar A, et al. Hypertonic saline improves the LCI in paediatric CF patients with normal lung function. Thorax 2010; 65: 379-383.

41 Green K, Buchvald FF, Marthin JK, et al. Ventilation inhomogeneity in children with primary ciliary dyskinesia. Thorax 2011; 67: 49-53.

42 Gronkvist M, Bergsten E, Gustafsson PM. Effects of body posture and tidal volume on inter- and intraregional ventilation distribution in healthy men. J Appl Physiol 2002; 43: 634-642.

43 Schibler A, Schneider M, Frey U, et al. Moment ratio analysis of multiple breath nitrogen washout in infants with lung disease. Eur Respir J 2000; 15: 1094-1101.

44 Fuchs SI, Buess C, Lum S, et al. Multiple breath washout with a sidestream ultrasonic flow sensor and mass spectrometry: a comparative study. Pediatr Pulmonol 2006; 41: 1218-1225.

45 Fuchs SI, Gappa M. Helium cannot simply be exchanged for SF6 in multiple breath washout. Eur Respir J 2009; 34: Suppl. 53, 204s.

46 Pillow JJ, Ljungberg H, Hülskamp G, et al. Functional residual capacity measurements in healthy infants: ultrasonic flow meter versus a mass spectrometer. Eur Respir J 2004; 23: 763-768.

47 Schibler A, Hall GL, Businger F, et al. Measurement of lung volume and ventilation distribution with an ultrasonic flow meter in healthy infants. Eur Respir J 2002; 20: 912-918.

48 Latzin P, Sauteur L, Thamrin C, et al. Optimized temperature and deadspace correction improve analysis of multiple breath washout measurements by ultrasonic flowmeter in infants. Pediatr Pulmonol 2007; 42: 888-897.

49 Mauritz KA, Moore RB. State of understanding of nafion. Chem Rev 2004; 104: 4535-4585.

50 Fuchs SI, Eder J, Ellemunter $\mathrm{H}$, et al. Lung clearance index: normal values, repeatability, and reproducibility in healthy children and adolescents. Pediatr Pulmonol 2009; 44: 1180-1185.

51 Macleod KA, Horsley AR, Bell NJ, et al. Ventilation heterogeneity in children with well controlled asthma with normal spirometry indicates residual airways disease. Thorax 2009; 64: 33-37.

52 Pittman JE, Johnson RC, Jones PW, et al. Variability of a closed, rebreathing setup for multiple breath wash-out testing in children. Pediatr Pulmonol 2012; 47: 1242-1250.

53 Kraemer R, Baldwin DN, Ammann RA, et al. Progression of pulmonary hyperinflation and trapped gas associated with genetic and environmental factors in children with cystic fibrosis. Respir Res 2006; 7: 138.

54 Larsson A, Jonmarker C, Werner O. Ventilation inhomogeneity during controlled ventilation. Which index should be used? J Appl Physiol 1988; 65: 2030-2039.

55 Pillow JJ, Frerichs I, Stocks J. Lung function tests in neonates and infants with chronic lung disease: global and regional ventilation inhomogeneity. Pediatr Pulmonol 2006; 41: 105-121.
56 Verbanck S, Paiva M, Schuermans D, et al. Relationships between the lung clearance index and conductive and acinar ventilation heterogeneity. J Appl Physio 2012; 112: 782-790.

57 Bouhuys A, van Lennep $H$. Effect of body posture on gas distribution in the lungs. J Appl Physiol 1962; 17: 38-42.

58 Saatci E, Miller DM, Stell IM, et al. Dynamic dead space in face masks used with noninvasive ventilators: a lung model study. Eur Respir J 2004; 23: 129-135.

59 Habib RH, Lutchen KR. Moment analysis of a multibreath nitrogen washout based on an alveolar gas dilution number. $A m$ Rev Respir Dis 1991; 144: 513-519.

60 Schulzke SM, Hall GL, Nathan EA, et al. Lung volume and ventilation inhomogeneity in preterm infants at 15-18 months corrected age. J Pediatr 2010; 156: 542-549.e2.

61 Saidel GM, Salmon RB, Chester EH. Moment analysis of multibreath lung washout. J Appl Physiol 1975; 38: 328-334.

62 Haidopoulou K, Lum S, Turcu S, et al. Alveolar LCI vs. standard LCI in detecting early CF lung disease. Respir Physiol Neurobiol 2012; 180: 247-251.

63 Fowler WS. Lung function studies; the respiratory dead space. Am J Physiol 1948; 154: 405-416.

64 Langley F, Even P, Duroux P, et al. Conséquences ventilatoires de l'occlusion unilatérale de l'artère pulmonaire [Ventilatory consequences of unilateral pulmonary artery occlusion]. Les Colloques des 1'Institut National de la Santé et de la Recherche Médicale 1975; 51: 209-212.

65 Saniie J, Saidel GM, Chester EH. Real-time moment analysis of pulmonary nitrogen washout. J Appl Physiol 1979; 46: 1184-1190.

66 Robinson PD, Lindblad A, Gustafsson PM. Comparison of the utility of multiple breath inert gas washout parameters in cystic fibrosis. Thorax 2010; 65: 659 .

67 Paiva M. Two new pulmonary functional indexes suggested by a simple mathematical model. Respiration 1975; 32: 389-403.

68 Paiva M, van Muylem A, Engel LA. Slope of phase III in multibreath nitrogen washout and washin. Bull Eur Physiopathol Respir 1982; 18: 273-280.

69 Dutrieue B, Vanholsbeeck F, Verbanck S, et al. A human acinar structure for simulation of realistic alveolar plateau slopes. J Appl Physiol 2000; 89: 1859-1867.

70 Paiva M, Engel LA. Model analysis of gas distribution within human lung acinus. J Appl Physiol 1984; 56: 418-425.

71 Verbanck S, Paiva M. Model simulations of gas mixing and ventilation distribution in the human lung. J Appl Physiol 1990; 69: 2269-2279.

72 Verbanck S, Paiva M. Gas mixing in the airways and airspaces. Compr Physiol 2011; 1: 835-882.

73 Cumming G, Horsfield K, Jones JG, et al. The influence of gaseous diffusion on the alveolar plateau at different lung volumes. Respir Physiol 1967; 2: 386-398.

74 Jones JG. The effect of preinspiratory lung volume on the result of the single breath $\mathrm{O}_{2}$ test. Respir Physiol 1967; 2: 375-385.

75 Crawford $\mathrm{AB}$, Cotton DJ, Paiva $\mathrm{M}$, et al. Effect of airway closure on ventilation distribution. J Appl Physiol 1989; 66: 2511-2515.

76 Crawford $\mathrm{AB}$, Cotton DJ, Paiva M, et al. Effect of lung volume on ventilation distribution. J Appl Physiol 1989; 66: 2502-2510.

77 Lacquet LM, van Muylem A. He and SF6 single-breath expiration curves. Comparison with the Paiva-Engel model Bull Eur Physiopathol Respir 1982; 18: 239-246.

78 Crawford AB, Makowska M, Engel LA. Effect of tidal volume on ventilation maldistribution. Respir Physiol 1986; 66: 11-25.

79 West JB, Hugh-Jones P. Pulsatile gas flow in bronchi caused by the heart beat. J Appl Physiol 1961; 16: 697-702.

80 Stuart-Andrews CR, Kelly VJ, Sands SA, et al. Automated detection of the phase III slope during inert gas washout testing. J Appl Physiol 2012; 112: 1073-1081.

81 Engel LA, Grassino A, Anthonisen NR. Demonstration of airway closure in man. J Appl Physiol 1975; 38: 1117-1125. 
82 Leblanc P, Ruff F, Milic-Emili J. Effects of age and body position on "airway closure" in man. J Appl Physiol 1970; 28: 448-451.

83 Benedik PS, Baun MM, Keus L, et al. Effects of body position on resting lung volume in overweight and mildly to moderately obese subjects. Respir Care 2009; 54: 334-339.

84 Gustafsson PM, Johansson HJ, Dahlback GO. Pneumotachographic nitrogen washout method for measurement of the volume of trapped gas in the lungs. Pediatr Pulmonol 1994; 17: 258-268.

85 Gustafsson PM, Kallman S, Ljungberg H, et al. Method for assessment of volume of trapped gas in infants during multiplebreath inert gas washout. Pediatr Pulmonol 2003; 35: 42-49.

86 Hughes JM, Rosenzweig DY, Kivitz PB. Site of airway closure in excised dog lungs: histologic demonstration. J Appl Physiol 1970; 29: 340-344.

87 Burger EJ Jr, Macklem P. Airway closure: demonstration by breathing 100 percent $\mathrm{O}_{2}$ at low lung volumes and by $\mathrm{N}_{2}$ washout. J Appl Physiol 1968; 25: 139-148.

88 Milic-Emili J, Torchio R, D'Angelo E. Closing volume: a reappraisal (1967-2007). Eur J Appl Physiol 2007; 99: 567-583.

89 Sixt R, Bake B, Oxhoj H. The single-breath N2-test and spirometry in healthy non-smoking males. Eur J Respir Dis 1984; 65: 296-304.

90 Michaelson ED, Wanner A, Watson H, et al. Distribution of ventilation in normal children. Chest 1979; 76: 658-662.

91 Hankinson JL. Computer determined closing volumes. Comput Biomed Res 1977; 10: 247-257.

92 Ljungberg HK, Gustafsson PM. Peripheral airway function in childhood asthma, assessed by single-breath $\mathrm{He}$ and $\mathrm{SF}_{6}$ washout. Pediatr Pulmonol 2003; 36: 339-347.

93 Van Muylem A, Baran D. Overall and peripheral inhomogeneity of ventilation in patients with stable cystic fibrosis. Pediatr Pulmonol 2000; 30: 3-9.

94 Van Muylem A, Verbanck S, Estenne M. Monitoring the lung periphery of transplanted lungs. Respir Physiol Neurobiol 2005; 148: 141-151.

95 Olfert IM, Prisk GK. Effect of 60 degrees head-down tilt on peripheral gas mixing in the human lung. J Appl Physiol 2004; 97: 827-834.

96 Lundin G. Nitrogen elimination during oxygen breathing. Acta Physiol Scand Suppl 1953; 111: 130-143.

97 Kjellman B. Ventilatory efficiency, capacity and lung volumes in healthy children. Scand J Clin Lab Invest 1969; 23: 19-29.
98 Ostlund A, Sporrong A, Linnarsson D, et al. Effects of sulphur hexafluoride on psychomotor performance. Clin Physiol 1992; 12: 409-418.

99 Houghton JT, Ding Y, Griggs DJ, et al. Climate Change 2001. Cambridge, Cambridge University Press, 2001.

100 Schulzke SM, Deeptha K, Sinhal S, et al. Nasal versus face mask for multiple-breath washout technique in preterm infants. Pediatr Pulmonol 2008; 43: 858-865.

101 Verbanck S, Schuermans D, Van Muylem A, et al. Ventilation distribution during histamine provocation. J Appl Physiol 1997; 83: 1907-1916.

102 Downie SR, Salome CM, Verbanck S, et al. Ventilation heterogeneity is a major determinant of airway hyperresponsiveness in asthma independent of airway inflammation. Thorax 2007; 62: 684-689.

103 Sigurs N, Aljassim F, Kjellman B, et al. Asthma and allergy patterns over 18 years after severe RSV bronchiolitis in the first year of life. Thorax 2010; 65: 1045-1052.

104 Salamon E, Gain K, Hall GL. Wait time effect on repeat multiple breath nitrogen washout FRC. Eur Respir J 2010; 36, 54: 656s.

105 Aurora P, Kozlowska W, Stocks J. Gas mixing efficiency from birth to adulthood measured by multiple-breath washout. Respir Physiol Neurobiol 2005; 148: 125-139.

106 Wanger J, Clausen JL, Coates A, et al. Standardisation of the measurement of lung volumes. Eur Respir J 2005; 26: 511-522.

107 Newth CJ, Enright P, Johnson RL. Multiple-breath nitrogen washout techniques: including measurements with patients on ventilators. Eur Respir J 1997; 10: 2174-2185.

108 Robinson PD, Stocks J, Aurora P, et al. Abbreviated multi-breath washout for calculation of lung clearance index. Pediatr Pulmonol 2012 [in press DOI: 10.1002/ppul.22618].

109 Gustafsson PM. Pulmonary gas trapping increases in asthmatic children and adolescents in the supine position. Pediatr Pulmonol 2003; 36: 34-42.

110 Latzin P, Roth S, Thamrin C, et al. Lung volume, breathing pattern and ventilation inhomogeneity in preterm and term infants. PLoS One 2009; 4: e4635.

111 Hutten GJ, van Eykern LA, Latzin P, et al. Relative impact of respiratory muscle activity on tidal flow and end expiratory volume in healthy neonates. Pediatr Pulmonol 2008; 43: 882-891.

112 Singer F, Stern G, Thamrin C, et al. Tidal volume single breath washout of two tracer gases-a practical and promising lung function test. PloS One 2011; 6: e17588. 